\title{
Picrasinoside H
}

National Cancer Institute

\section{Source}

National Cancer Institute. Picrasinoside H. NCI Thesaurus. Code C1771.

A quassinoid glycoside phytochemical isolated from the plant Picrasma ailanthoides with potential antineoplastic activity. (NCI04) 